\title{
A Framework for Simulating Turbine-Based Combined- Cycle Inlet Mode-Transition
}

\author{
Dzu K. Le ${ }^{1}$, Daniel R. Vrnak ${ }^{2}$, John W. Slater ${ }^{3}$, and Emil O. Hessel ${ }^{4}$ \\ NASA Glenn Research Center, Cleveland, Ohio, 44135 USA
}

\begin{abstract}
A simulation framework based on the Memory-Mapped-Files technique was created to operate multiple numerical processes in locked time-steps and send $\mathrm{I} / \mathrm{O}$ data synchronously across to one-another to simulate system-dynamics. This simulation scheme is currently used to study the complex interactions between inlet flow-dynamics, variable-geometry actuation mechanisms, and flow-controls in the transition from the supersonic to hypersonic conditions and vice-versa. A study of Mode-Transition Control for a high-speed inlet windtunnel model with this MMF-based framework is presented to illustrate this scheme and demonstrate its usefulness in simulating supersonic and hypersonic inlet dynamics and controls or other types of complex systems.
\end{abstract}

\section{Nomenclature}

$B C=$ Flow boundary-condition (upstream or at inlet exit)

$d t \quad=$ Time differential

$F=$ generalized-force on the LS Path variable ramp sections, with generalized-coordinate for rigid-body ramp motions being the stroke of the main actuator of the LS Path ramp

HS Path = High-speed flow path (inlet flow-path for the ramjet/scramjet engine)

LS Path = Low-speed flow path (inlet flow-path for the turbine engine)

$\mathcal{M} \quad=$ Stroke-dependent generalized-mass of the LS Path variable ramp sections

$V A=$ "Vertical actuators" - i.e., the two side-actuators besides the main actuator of the LS Path ramp

$x \quad=\quad$ Stroke of the main-actuator of the LS Path ramp

\section{Introduction}

$\mathrm{T}$ HIS work is motivated by the current need for system-level dynamics simulations in High-Speed-Inlet ModeTransition Control research at NASA for Turbine-Based Combined-Cycle (TBCC) propulsion. ${ }^{1,2}$ Inlet controlsystem design and Mode-Transition control development can benefit from dynamic simulations at different levels of fidelity and complexity. System-level dynamics simulation is needed to understand the complex interactions of airinlet dynamics, propulsion engines, control-effectors, and feedback sensors. A versatile modeling framework using the Memory-Mapped Files (MMF) technique was created for this purpose. It allows for separate simulationprocesses running simultaneously on a single desktop computer in locked time-steps (i.e., synchronous stepping of simulated time) to exchange $I / O$ data simulating system-dynamics and interactions. ${ }^{7,8}$

The inlet in this simulation study of Mode-Transition control represents a large-scale wind-tunnel model of a hypersonic-inlet (about 30-foot long) which was designed for inlet testing and control research in a supersonic wind-

${ }^{1}$ Aerospace Engineer, Propulsion and Control Systems Analysis, MS 86-11, 21000 BrookPark Rd., Cleveland, Ohio, 44135 USA.

${ }^{2}$ Computer Engineer, Propulsion and Control Systems Analysis, MS 86-11, 21000 BrookPark Rd., Cleveland, Ohio, 44135 USA.

${ }^{3}$ Aerospace Engineer, Inlet and Nozzle Branch, MS 5-12, 21000 BrookPark Rd., Cleveland, Ohio, 44135 USA, AIAA Senior Member.

${ }^{4}$ Georgia Institute of Technology Student-Trainee, Mission Design and Analysis, MS 105-3, 21000 BrookPark Rd., Cleveland, Ohio, 44135 USA. 
tunnel at NASA Glenn. ${ }^{3,4}$ This inlet concept is depicted by Figure 1 which features two parallel flow-paths: one, named the Low-Speed Path ("LS Path"), is to provide air-inlet for a turbine engine in subsonic and supersonic flight up to Mach 4; and another, the High-Speed Path ("HS Path"), for a dual-mode ramjet and scramjet engine in flight regions above Mach $4 .^{9}$ The forward section of each flow path features mixed-compression geometry with a movable cowl. The LS Path is shown as the upper flow path; the HS Path is the lower one. For this study, nominal mode transition is to occur at Mach 4 free-stream condition.

At mode transition, the splitter (the cowl of the LS Path) is rotated to cocoon the LS Path. ${ }^{9}$ During modetransition, the ramp of the LS Path can be raised or lowered using three hydraulic actuators located at the end of the second ramp section. The main flow-control elements for inlet performance and stability during mode transition consist of (passive) air-bleeds at critical locations along the ramp, sidewall, and cowl surfaces, and four air-bypass doors for active-control of air bypassing the turbine through a plenum located near the aft end of the diffuser. The air-bypass doors are crucial for maintaining a favorable pressure and mass-flow recovery and keeping the normal shock downstream from the inlet throat in the LS Path.

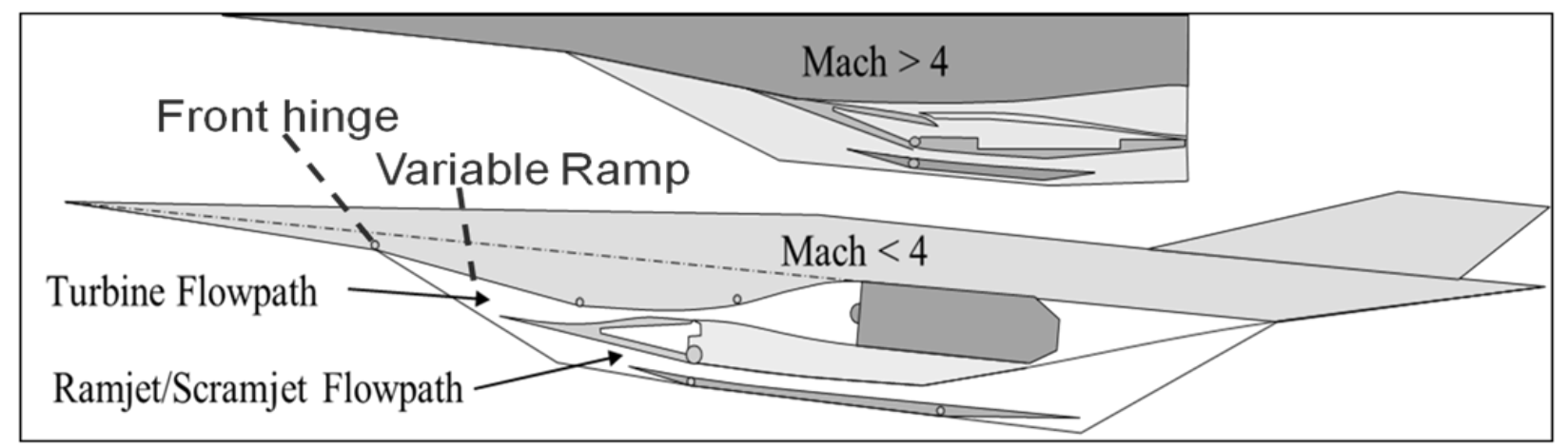

Figure 1. A conceptual Hypersonic Propulsion system.

The inlet-system simulation that employed the MMF technique includes the following parts: (a) A onedimensional Computational Fluid Dynamics (CFD) model of compressible air-flow through the LS Path; (b) A high-fidelity simulation model of inlet servo-mechanical dynamics and actuation controls; (c) Computation of aerodynamic loads on the LS Path cowl exterior-surface and on both sides of the HS Path cowl, using tabulated aero-coefficient data based-on a two-dimensional aerodynamics CFD model ${ }^{10}$; (d) A simulation model of the bypass door controls and possible effects of plenum dynamics on inlet exit flow or on turbine and inlet couplings.

The simulation of inlet mode transition control using the MMF-technique will be presented in Section II, including some details in modeled component dynamics. Simulation study of system-dynamics behaviors, control authority, and inlet mode-transition performance and implications will be presented in Section III. Lastly, a conclusion about this simulation framework and the control study will be given in Section IV.

\section{Inlet Mode-Transition Simulation in an MMF-Framework}

The implemented MMF-framework for simulating mode transition control-dynamics for high-speed inlets of the type shown in Figure 1 will be described in more details in this Section. The inlet system to be simulated ${ }^{3,4}$ is outlined in Figure 2.

\section{A. Simulation-Scheme Overview}

The MMF-simulation scheme is outlined in Figure 3, in which the "MMF Interface" is a function that keeps the following two separate (non-real-time) computational processes running simultaneously in locked time-steps with data exchange both ways to simulate dynamics interactions: (1) A one-dimensional (1-D) CFD model of Inlet FlowDynamics of the LS Path; and, (2) A high-fidelity Servo-Mechanical Control model consisting of various interconnected sub-modules mainly for simulating inlet-mechanics and actuator control-dynamics.

The "Servo-Mechanical" side (i.e., the computational process \#2) in this setup of parallel simulations includes a subroutine for calculating Aero loads on the LS Path variable ramp sections and the LS Path cowl based on "Inlet Flow-Dynamics" calculations of static-pressure distribution along the LS Path. The calculation of aero-loads on the HS Path cowl and on the exterior surface of the LS Path cowl (see Figure 2) is based on tabulated aerodynamiccoefficients pre-calculated by a two-dimensional aerodynamics CFD model. The "Servo-Mechanical" side of this 
simulation also includes a subroutine to simulate air-bypass control, and a dynamic model for the boundary-conditions of the LS Path exit-flow.

Consequently, the outputs of "Inlet Flow-Dynamics" are affected by many variables of the "Servo-Mechanical Model" side; such as, the flow-path geometry variables, the air-bypass doors exit area, and the LS Path exitflow boundary-condition (i.e., static-pressure at the exit).

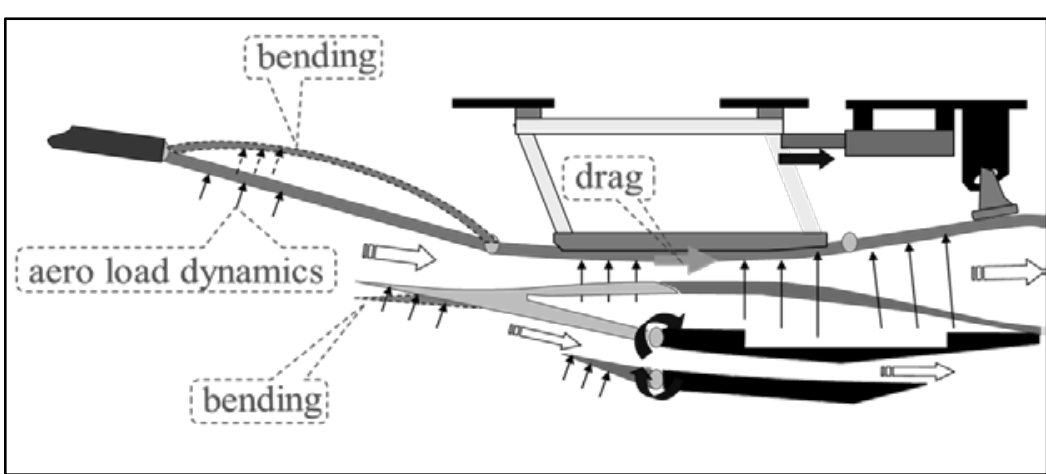

Figure 2. Illustration of inlet Variable-Geometry and Actuation.

NASA's FORTRAN 77 based legacy code of a one-dimensional CFD model named "Large Perturbation Inlet (LAPIN)" ${ }^{5,6}$ is used for modeling air-flow dynamics in the LS Path. The LAPIN includes options for modeling airbleeds and bypasses, flow-path variable-geometry, and various types of disturbances and downstream BC's.

The LAPIN executable is arranged to run separately within a Microsoft Disk Operating System (MS-DOS®) Command Window. Data exchanges in locked time-steps at high-rate (every 40 milliseconds simulated time interval) between this LAPIN process and the "Servo-Mechanical" simulation are handled by MMFinterface codes and intercepts imbedded within LAPIN. ${ }^{7}$

Let us briefly review the application software that was used for implementing this MMF technique. The MMF application software was developed to support computer programs written and compiled in C, $\mathrm{C}++, \mathrm{C \#}$ and Visual Basic languages, but not for the FORTRAN language. So, the MMF implementation $\mathrm{C}++$ subroutines were composed and compiled to create compatible object files for linking with the FORTRAN compiled object files. Using this approach, the MMF subroutines were imbedded in LAPIN to be invoked at various points in the main simulation loop of LAPIN for data exchanges

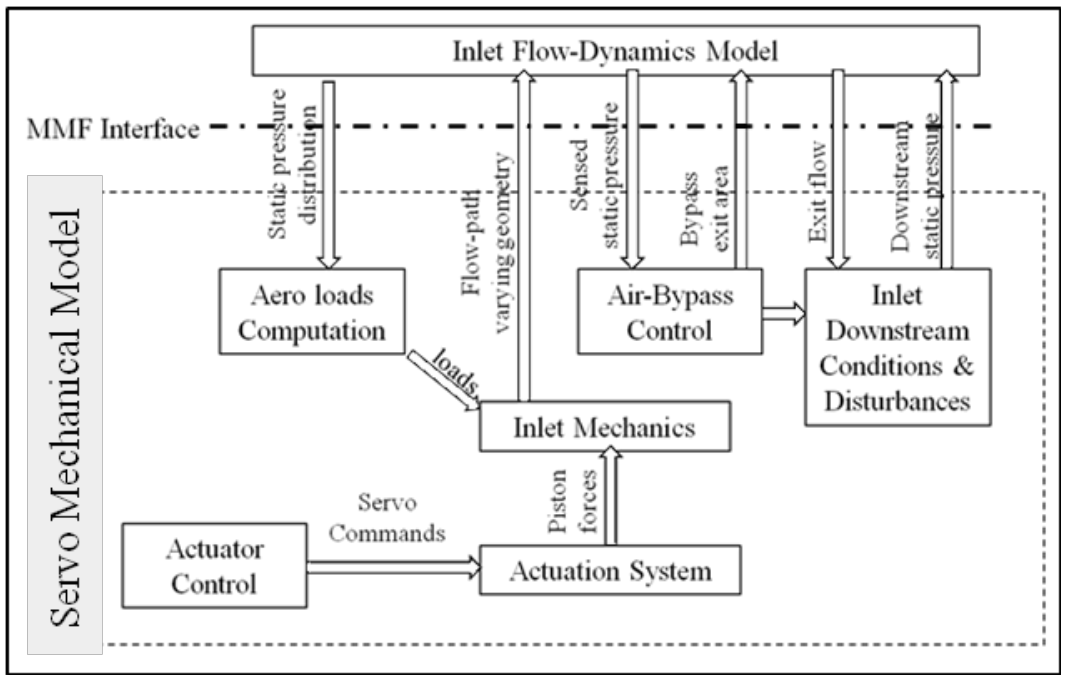

Figure 3. Overview of the MMF-simulation of Inlet Mode-Transition Control -- Two separate, interactive computation processes: A simulation model of Inlet "Servo-Mechanical Control" (dotted box), and a 1D-CFD model of Inlet Flow-Dynamics.

with the process simulating the Servo-Mechanical control aspects. Microsoft (MS) Visual $C$ was also used to compile the MMF interface codes on the "Servo-Mechanical" side of the simulation for connection with the LAPIN process. Interested readers are referred to Vrnak D. et.al. ${ }^{7}$ for more details about the particular MMF techniques implemented.

\section{B. Math-Formulation of Component-Dynamics}

Potential interactions between inlet structural-compliances and flex-dynamics on the one side, and actuators dynamics and flow-dynamics on the other can also be included in the simulation. At this point, the simulation of inlet ramp and cowls motions are limited to just rigid-body dynamics.

Note that the LS Path ramp system consists of three variable sections adjacent to a non-moving pre-compressionramp surface (which is to replicate the effect of a fore-body on free-stream air): the rotating first section, ramp section 1, with its rotating surface situated at the end of the pre-compression ramp (see Figures 1 and 2); the midsection, ramp section 2, supported by linkage bars (four) off its moving base on rails; and, the end section, ramp section 3, which is also the upper surface of the LS Path diffuser. 
These LS Path variable-ramp sections are operated by three hydraulic actuators: a main actuator parallel to the rails to push or pull at the moving base of the ramp to raise or lower the ramp with the support linkages; and two Vertical Actuators (VA's) to push or pull against the underside end of ramp section 2. The VA's push against the ramp section 2 in tandem with the pulling-in action of the main actuator to raise the ramp towards the LS Path cowl surface to reduce the LS Path throat height. [Throat height reduction is required when the flight-vehicle accelerates from lower speeds towards the Mach 4 flight condition. ${ }^{9}$ ] Conceptually, depending on vehicle and mission design, TBCC mode-transition from turbine to ramjet/scramjet phases can occur across the Mach-number range 3 to 4 . $^{9}$

Since only Rigid-Body motions are considered here, math formulation of the LS Path ramp equation of motions and actuators dynamics in the setting of the wind-tunnel can be done in the Lagrangian Mechanics approach using the stroke of the main actuator, denoted " $x$ ", as the single generalized coordinate. Specifically, the kinetic energy $K_{\mathrm{e}}$ of the ramp motion relative to the inlet Structural Reference frame (SR-frame) is expressible in the following form:

$$
K_{e}=\frac{1}{2} \mathcal{M}(x)(d x / d t)^{2}
$$

The stroke-dependent generalized mass of the combined moving parts of the ramp, denoted $\mathcal{M}(s)$, is defined by:

$$
\mathcal{M}(x) \equiv I_{1}\left(\frac{d \theta_{1}}{d x}\right)^{2}+M_{2}\left(\left(\frac{d x_{2}}{d x}\right)^{2}+\left(\frac{d y_{2}}{d x}\right)^{2}\right)+M_{3}\left(\left(\frac{d x_{3}}{d x}\right)^{2}+\left(\frac{d y_{3}}{d x}\right)^{2}\right)+M_{b}+I_{3}\left(\frac{d \theta_{3}}{d x}\right)^{2}
$$

The parameters and variables in expression (2) are defined as follows: " $I_{\mathrm{i}}$ " is the moment of inertia of ramp section $i$ (for $i=1,3$ ) about the respective front hinge; " $M_{\mathrm{i}}$ ", mass of ramp section $i$ (for $i=2,3$ ); " $M_{\mathrm{b}}$ ", mass of the moving base of ramp section 2; $\left(x_{2}(x), y_{2}(x)\right)$, the position abscissa and ordinate of ramp section 2 as function of " $x$ "; $\left(x_{3}(x), y_{3}(x)\right)$, position of ramp section 3 Center-of-Mass $(C M)$; and, $\theta_{\mathrm{i}}(\mathrm{x})$, the inclination angle of ramp section $i$ (for $i=1,3$ ) relative to the $\mathrm{X}$-axis of the $S R$-frame. The origin of the $S R$-frame is chosen to be fixed at the precompression ramp leading-edge (The pre-compression ramp extends from that edge to the front hinge of ramp section 1); the SR-frame $x$-axis is parallel to the moving base of the four-linkage support of ramp section 2 and pointing downstream; the $Y$-axis is pointing towards the ramp surface.

Consequently, if $F(x, t)$ denotes the total generalized force on this variable ramp (which is dependent on both stroke and time; i.e., on $x$ and $t$ ), the ramp motion is defined by the following Lagrangian-Mechanics equation, in which the upper dot symbol (") on " $x$ " stands for the time-derivative operator on " $x$ ":

$$
\mathcal{M}(x) \ddot{x}+\frac{1}{2} \frac{d \mathcal{M}(x)}{d x} \dot{x}^{2}=F(x, t)
$$

As defined above $E q(3), F(x, t)$ must include the contribution of actuator forces (both the main actuator and the two VA's), and also all other external forces on all the moving parts of the LS Path ramp -- such as, aerodynamic loading, seal frictions (including static, viscous, and Coulomb friction effects), and gravity (as in the wind-tunnel test environment, in which the SR-frame is considered inertial). [Note: This formulation is also applicable for simulating inlet mechanics in the flight environment, provided a term capturing thrust acceleration effects is also included in $F(x, t)]$.

Aero-loading on the moving parts of the ramp are approximated from LAPIN static pressure calculations in the LS Path -- which is received by the "Servo-Mechanical" side of the MMF scheme by the connection-block named "From_LAPIN" shown in Figure 4. These physical forces and moments are then converted to generalized forces based on the relation between the stroke, "x", and the position of the various variable parts of the ramp -- base, linkages, and sections. For example, considering the ramp section 1, the effects of aero-loads, seal frictions, and gravity are calculated from their total moments about the pivoting axis. The contribution to $F(x, t)$ by these effects, as moments about the pivoting axis, is computed using the same conversion factor " $(\pi / 180) \times d \theta_{1} / \mathrm{d} x$ "; where, " $d \theta_{1} / \mathrm{d} x$ " is the slope of the curve shown in Figure 5. This curve represents the relationship between the ramp inclination angle " $\theta_{1}$ " and " $\mathrm{x}$ ". On the other hand, since the constraint motion of the ramp section 2 is nonrotational; the contribution of aero-loads, frictions, and gravity on this part to $F(x, t)$ is computed simply by applying the factor " $d x_{2} / d x$ " or " $d y_{2} / d x$ " on the $x$-component or $y$-component, respectively, of the total of these forces.

The contribution of aero loads, frictions, and gravity on ramp section 3 to $F(x, t)$ is done similarly, and involves both the respective total force and total moment at the joint between ramp section 2 and section 3; therefore, the 
factor to convert physical forces and moments to generalized force in this case involves " $d x_{2} / d x$ ", " $d y_{2} / d x$ ", and " $d \theta_{3} / d x "$.

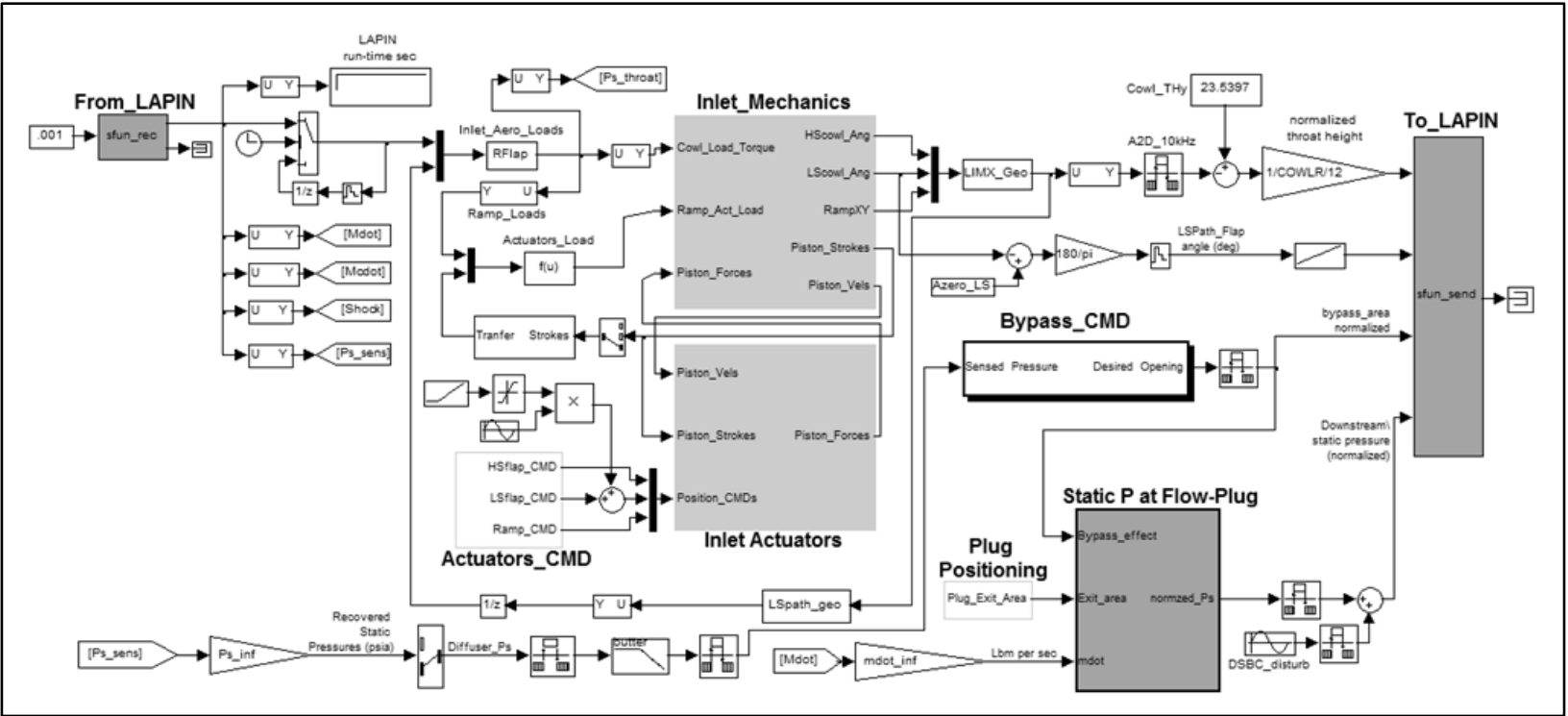

Figure 4. Top-level diagram of the "Servo-Mechanical Model" side of the MMF-scheme -- Ref. Figure 3

The conversion of the hydraulic forces of the VA's to the corresponding generalized-forces is done similarly using tabulated data for the geometric-constraint between the main mctuator stroke and the VA's stroke. For the sake of simplicity, further formulation details concerning generalized-forces computation will be skipped. The Lagrangian dynamics equations for the LS Path cowl and HS Path cowl are similarly derived using the respective actuator stroke as the generalized coordinate. These modeling components are simpler than in the ramp case, because they do not involve multiple hinged-sections.

The actuation system control-dynamics (for LS Path ramp, LS Path cowl, and HS Path cowl) are modeled with a fair amount of details, which includes servo-valve dynamics and control, and hydraulic cylinders and piston dynamics. In particular, the state variables " $x$ " and " $d x / d t$ " defined by Eq. (3) -- computed in the block "InletMechanics" in Figure 4 -- are used in the block "Inlet_Actuator" to model the effects of ramp piston motions on the hydraulic-fluid flows in the opposing volumes on either sides of the piston in each of the three cylinders (actuators) of the ramp.

The diagram shown in Figure 6 represents the simulated air-bypass control logic. The output of this module is the commanded total plenum exit area normalized by the square of the nominal height of the LS Path cowl leading edge. This control logic is essentially a digital Lead-Lag filter pair, with input from a pressure sensor located at the aft end of the diffuser. One digital filter is designed to lead by 45 degrees; and the other to lag by 45 degrees at the $15 \mathrm{~Hz}$ frequency. The purpose of the band-pass pre-filter (with the pass-band of 1 to $15 \mathrm{~Hz}$, see Figure 6) in series with these parallel Lead/Lag filters is to produce two resultant signals that are nearly orthogonal for time-localized signal contents of time-scales from 1 second to $1 / 15$ seconds. Consequently, any linear combination of the resultant signals will

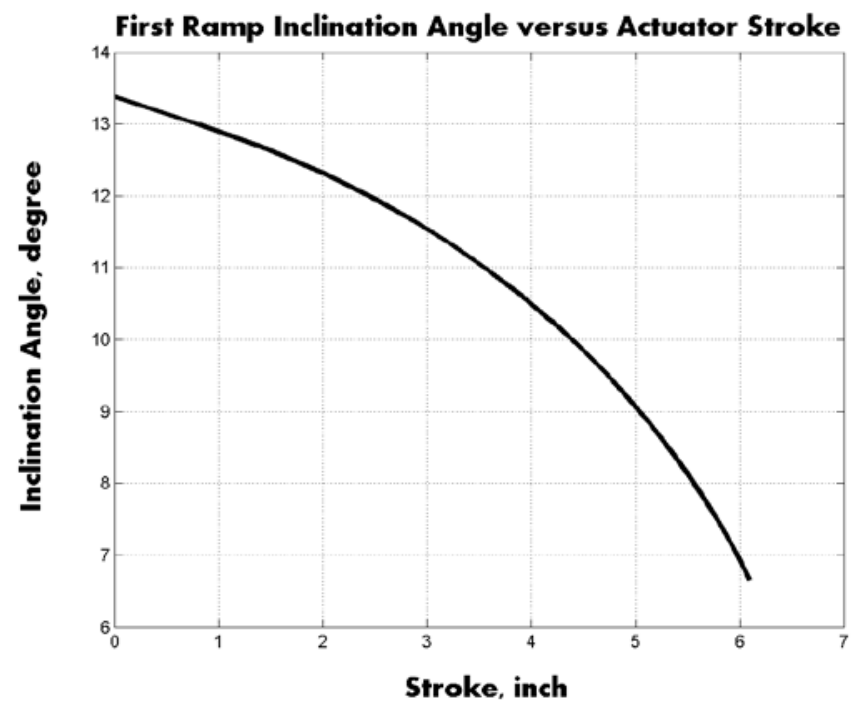

Figure 5. Variable-Geometry Actuation -- Relationship between the main actuator stroke, " $x$ ", and ramp section 1 inclination-angle 
capture such time-localized features in the input signal at any time, including their transient amplitude. ${ }^{11}$ This linear combination is also made with a certain phase lead (relative to the dominant features of $1 \mathrm{~Hz}$ to $15 \mathrm{~Hz}$ in the signal). The particular phase lead is numerically determined for optimal suppression of pressure oscillations below 25Hz. This is just a simple implementation of more complete schemes, in the Wavelet approach, for extracting nonlinear-transient features in feedback signals for vibration suppression. ${ }^{11}$

Also, the amplitude of the resultant signal is proportional to the transient (oscillation) amplitude of the input signal, using an adjustable gain. This resultant signal (a band-limited signal to use as feedback for oscillationsuppression) and an estimate of its amplitude are then added to a (scheduled or pre-set) mean-opening command for the bypass. Thus, the commanded opening of bypass plenum exit area would be adjusted proportionately with the sensed amplitude of oscillations. [Note: The command of mean-opening is numerically defined based on simulation results. Theoretically, this can also be defined analytically, for example based on CFD analysis concerning flowdynamic sensitivity to bypass mean-flow.]

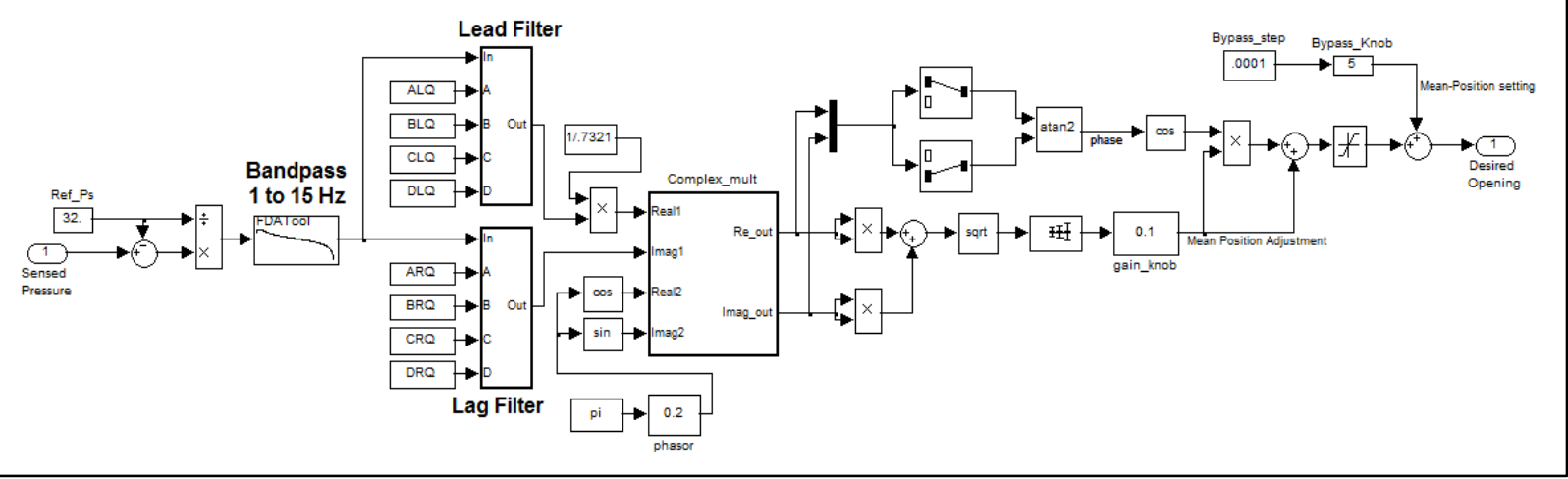

Figure 6. Block diagram of the Air-bypass Command calculation. -- Ref. Figure 4

Lastly, the LS Path exit flow BC is modeled as the static-pressure of an air-volume, with in-flow being the LS Path exit-flow, and the out flow is through a choked opening with adjustable area. This "volume dynamics" is essentially a parametric model to simulate the effect of the main mass-flow plug of the LS Path cold-pipe. The adjustable exit-area accounts for the adjustable position of the mass-flow plug. It will be shown that this exit -area can be scheduled to adjust the static pressure $B C$ of the LS Path exit-flow to keep the normal shock near the inlet throat. Augmenting the throat bleed system with the air bypass control is to provide additional stabilization for the normal-shock at critical operation conditions with the inlet precariously near 'unstart' (For a mixed-compression inlet, unstart is a transient phenomenon when the normal shock is violently expelled upstream from the throat to a position forward of the cowl lip).

\section{Inlet Mode-Transition Control and Dynamics Study}

A study of the inlet system dynamics behavior and mode-transition control as modeled by the MMF-based simulation scheme will be presented in this Section. First, simulated system dynamics behaviors responding to various control-effectors and to disturbances at flow exit will be studied. These simulation results will be the basis for the mode transition control strategy to be presented in the follow-on sub-section.

\section{A. Simulated Inlet Control-Dynamics Behaviors}

First, some simulation results will be presented in this sub-section to demonstrate the adequacy of this MMF simulation in representing inlet aerodynamics sensitivity to changes in flow-path geometry and to disturbances, and the effect of bleeds and bypasses. The purpose is also to study the following aspects of system behavior: (a) The sensitivity of inlet flow-dynamics (e.g., pressures, mass-flow rate, normal shock position, etc.) to exit-flow boundary condition s such as static-pressure; (b) The sensitivity of inlet flow-dynamics to LS Path cowl and ramp motions; and (c) The stabilization effect and control-authority (roughly below $20 \mathrm{~Hz}$ ) of air-bypass controls on the normalshock motions as well as on recovered flow pressures. These simulation cases are for the following free-stream airflow conditions: $\quad$ Free-stream static-pressure is 32.27lbf/ $\mathrm{ft}^{2}$ (psf); static-temperature, 178.57 degree Rankine; Mach number, 4.0; and ratio of specific-heat coefficients, $\gamma=1.40$. 
The simulation results in Figures 7 to 10 shows the effect of exit-flow BC, as static pressure, and disturbances (at the end of a cold pipe with a mass-flow plug) on both the calculated normal shock position and the staticpressure throughout the LS Path. If the LS Path cowl and ramp is commanded to hold a nominal position pertaining to a Mach 4 geometry, the relationship between the normal shock position and the exit-flow $B C$ represented as static pressure (normalized to the free-stream Total-Pressure, which is roughly 4900psf) is fairly piecewise-linear up to about 0.78 (see Figure 7). But, this relationship appears to reach a singularity when the pressure ratio reaches about 0.8 , where the sensitivity of the normal-shock position to back-pressure approaches infinity. Beyond this point it is practically impossible to push the normal shock closer to the LS Path throat without un-starting the inlet. Furthermore, since the sensitivity of the normal shock position is so great at this point, a slight perturbation in the $\mathrm{BC}$ can also result in an unstart. On the other hand, the relationship between exit normalized BC pressure and the normalized diffuser static pressure is linear all the way to the singularity point (see Figure 8). Understanding system dynamics sensitivities, such as the complexity shock-position sensitivity as seen in Figure 7 versus the linear relationship between inlet pressure-recovery and back-pressure in Figure 8 is vital for establishing realistic pressure recovery goals with acceptable and realistic stability margins.

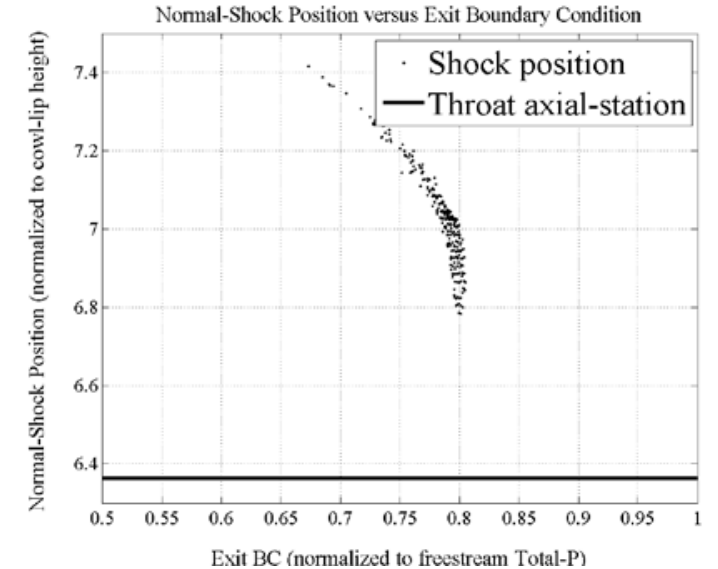

Figure 7. Effect of exit-flow BC on normal-shock position -- Simulated with nominal (zero-degree) cowl-angle command, air-bypass control-gain set at medium, and disturbance as $8 \mathrm{~Hz}$-variation in exit BC by \pm 1.4 percent.

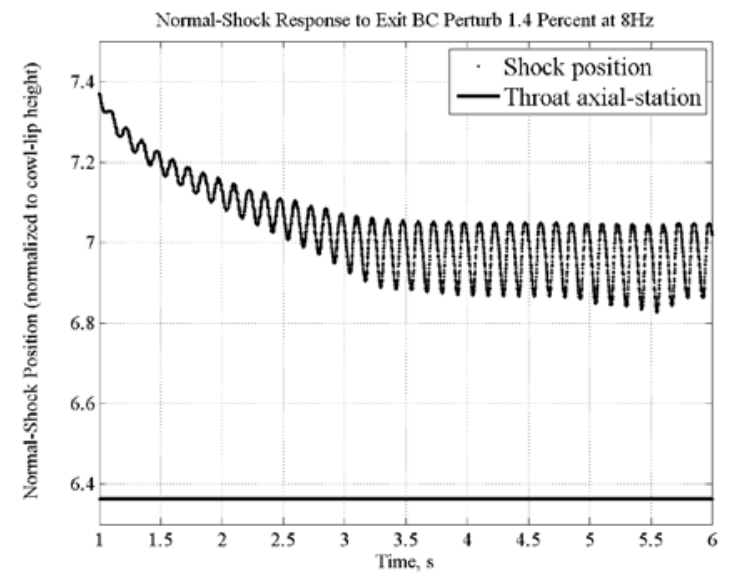

Figure 9. Flow sensitivity to disturbances -- Normalshock response to $8 \mathrm{~Hz}$-variations in exit $\mathrm{BC}$ by \pm 1.4 percent.

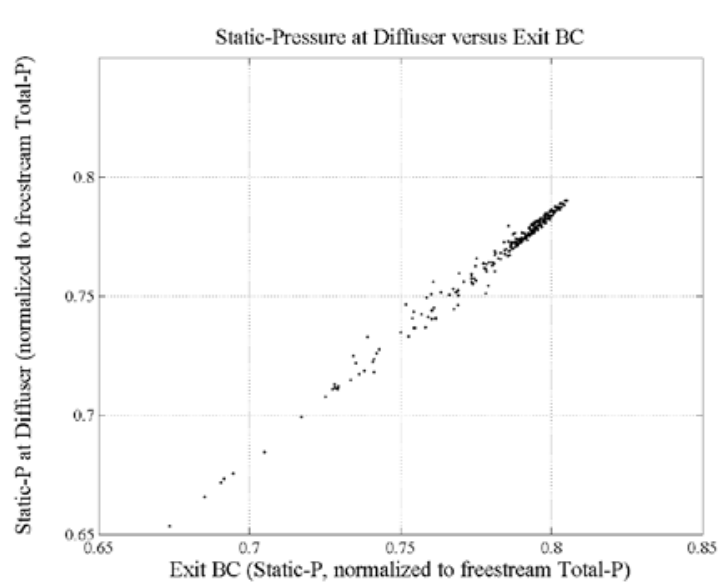

Figure 8. Effect of exit BC on static-pressure at the end of LS Path diffuser.

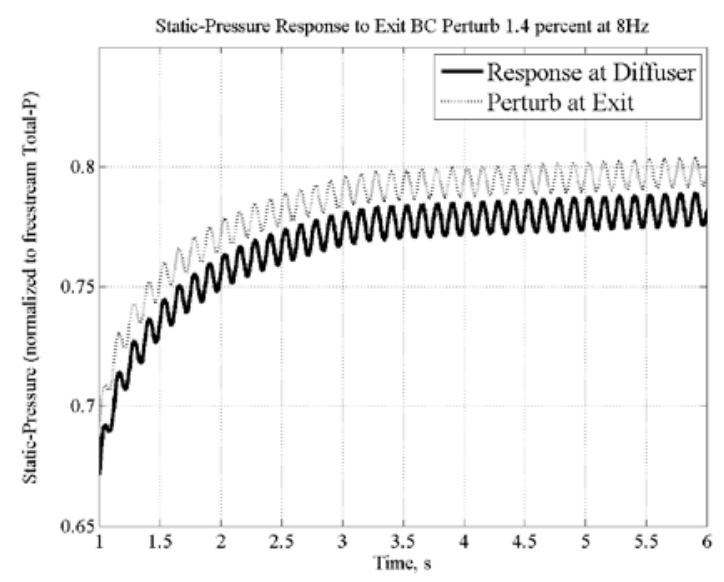

Figure 10. Flow sensitivity to disturbances -- Staticpressure response at LS Path diffuser to $8 \mathrm{~Hz}$-variations in exit BC by \pm 1.4 percent.

Examples of inlet-flow dynamics response to harmonic variations in the exit $B C$ is shown in Figures 9 and 10. These results are simulated without active bypass control. The simulation predicts that, as the shock moves closer to the throat its range of motion will increase even though the oscillations in $B C$ remain steady 
in amplitude and frequency. Meanwhile, the diffuser pressure amplitude remains linearly proportional to the disturbance amplitude.

The effect of open-loop position-commands for the LS Path cowl with harmonic waveforms on normal shock

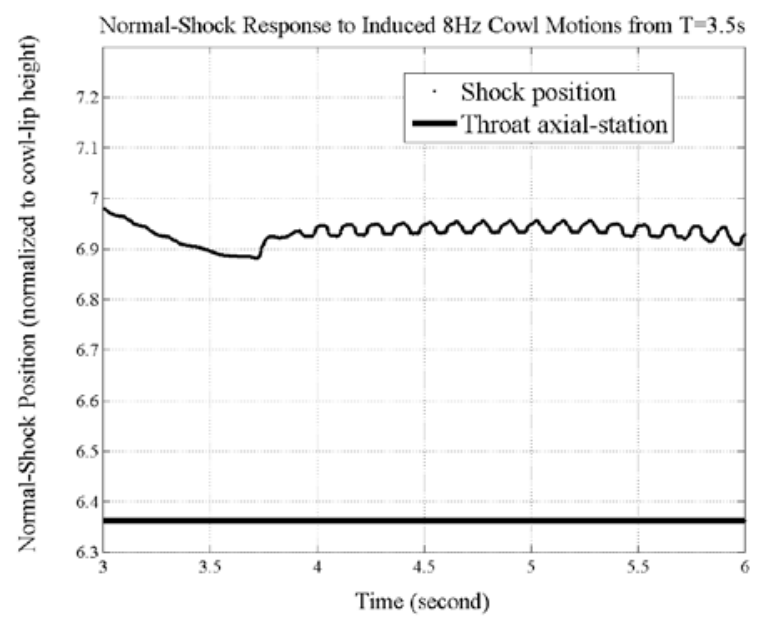

Figure 11. Flow sensitivity to Cowl motions --Normalshock response to cowl-position command with an $8 \mathrm{~Hz}-$ waveform (at T3.5 s). position is also significant as illustrated in Figure 11. The shift in normal-shock towards downstream shortly after the start of the harmonic command signals (harmonic commands started at $T=3.5 \mathrm{~s}$ ) is probably due to the slight change in mean cowl position due to the response of actuator to harmonics command near the hard-stop. Cowl rotation towards LS Path closing tends to push the normal shock downstream, while only inducing very small disturbances to static-pressures in the diffuser. Therefore, it is possible to take advantage of this effect to position the normal shock with cowl positioning, if necessary. Similar responses were recorded when disturbances were applied to the LS Path ramp position. These simulation results agree with known CFD results, that the LS Path normalshock position is sensitive to changes in ramp and cowl position ${ }^{10}$. Hence, it is necessary to fine-tune actuators control parameters to avoid servo oscillations and reduce the probability of inlet un-

start during mode transition.

The response of LS Path flow-dynamics to harmonic excitations with open-loop commands of air bypass plenum exit area is fairly strong (see Figures 12 and 13). The air bypass is employed to provide additional stability-margin for the normal-shock beyond what is passively available with volume dynamics and bleeds. Based on this simulation, the effective range of the bypass-control gain (Figure 6) to suppress pressure oscillations is from 0 to 0.3 . When this gain is set at Medium (i.e., about 0.1), the bypass control can only suppress static-pressure oscillations by roughly $30 \%$. Better suppression for oscillations below $15 \mathrm{~Hz}$ is attainable with higher gain values. However, it was found that higher gains would induce undesirable super-harmonics.

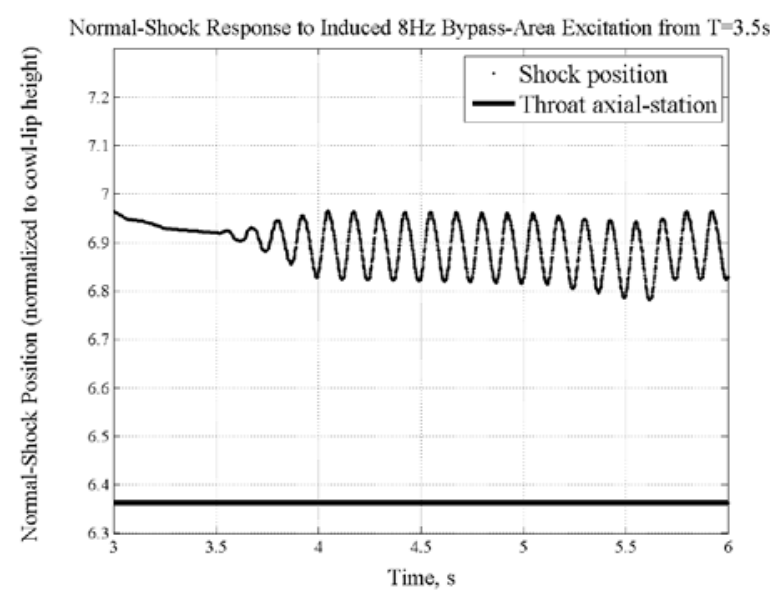

Figure 12. Air-bypass control-authority -- Normalshock response to air-bypass command with an $8 \mathrm{~Hz}$ waveform (at T3.5 s)

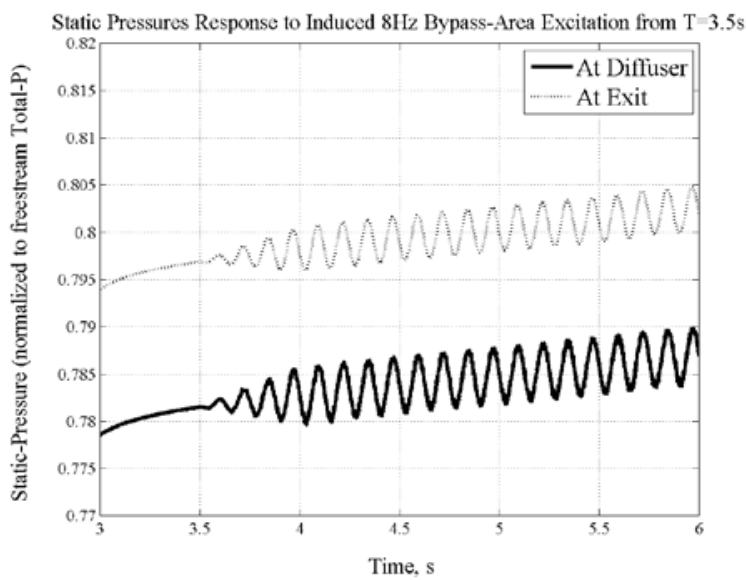

Figure 13. Air-bypass control-authority -- Staticpressure response at LS Path diffuser and flow-plug region to air-bypass cmd. (at T3.5 s) with an $8 \mathrm{~Hz}-$ waveform

\section{B. Mode-Transition Control Simulation}

To be presented next are some simulation results on Mach 4 mode transition control. As mentioned earlier, for the same $B C$ on exit-flow, the normal-shock tends to move downstream when the LS Path cowl is rotated towards closing. It will also be shown that, while the LS Path cowl is moved towards closing the sensitivity of the shock 
position near the "singular point (see Figure 7) is drastically reduced. Perhaps, this is because the aerodynamic throat of the LS path moves up stream when the LS cowl is closing. In this case, the shock can be pushed further upstream (than in the case of zero-degree cowl angle) by increasing the static-pressure on LS Path exit (by further closing the mass-flow plug). The best simulation result in this regard is attained when the air bypass control gain for active suppression of diffuser static-pressure is at a certain Medium value (A particular control-gain value, numerically derived, to result in roughly a $30 \%$ reduction in the oscillations level). Results from this case are shown in Figures 14 to 16.

Upon mode transition initiation, a particular schedule for LS Path flow-plug repositioning is also activated to increase the static-pressure of LS Path exit-flow (see the solid, thin line in Figure 15). The position of the plug can be scheduled to push the normal-shock upstream beyond the "singular point" (Comparing Figure 7 to Figure 16) while the LS Path cowl is rotating towards LS Path close-off (Figure 14) without unstarting the inlet. The normalshock moves forward and settles near the throat (roughly 4 inches downstream of the geometric throat) when the LS Path is halfway closed. As mentioned earlier, this is not possible if the LS Path cowl angle is fixed at zero-degree. These simulation results agree with CFD analyses ${ }^{10}$, that it is possible to achieve good stability and performance for inlet mode-transition with air-bypass control, combined with proper handling of inlet variable-geometry and

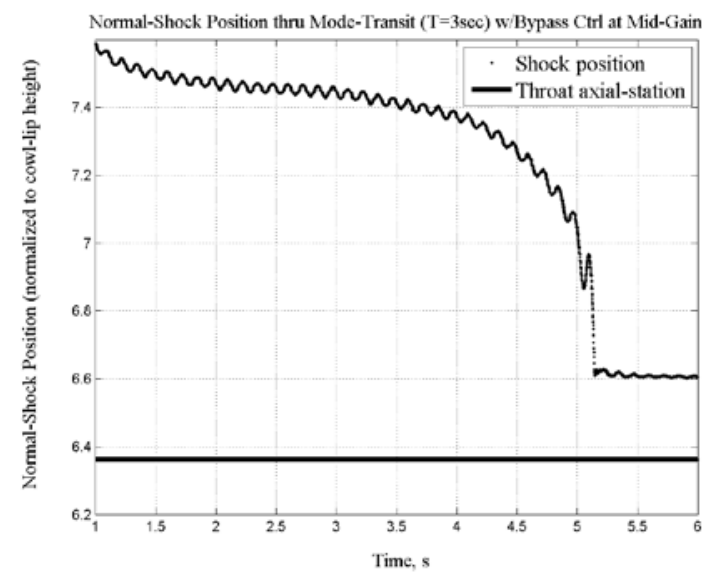

Figure 14. Normal-shock motions thru ModeTransition with Medium Air-bypass control gain, Cowl commanded to close to halfway from T3.5s to T5.0s, flow-plug position scheduled to keep the normal-shock at inlet throat.

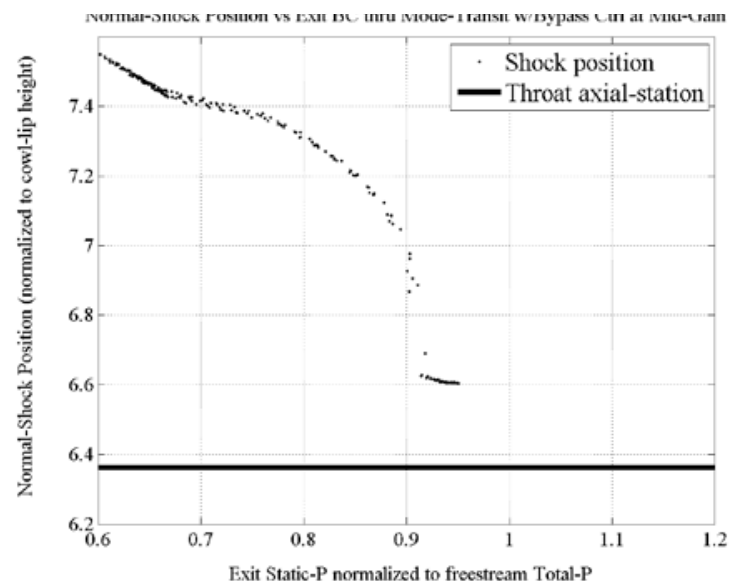

Figure 16. Effect of exit $B C$ on Normal-shock position thru Mode-Transition

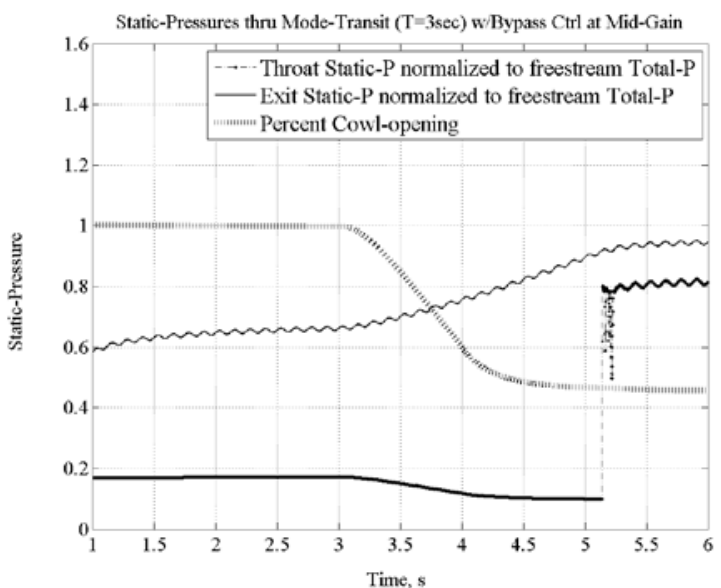

Figure 15. Static-pressure variations thru ModeTransition with Medium air bypass control gain

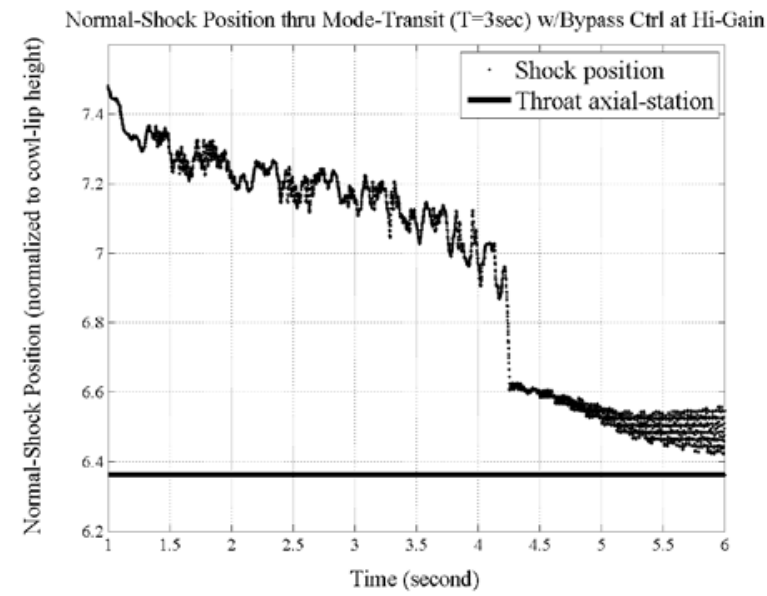

Figure 17. Normal-shock motions thru ModeTransition with air bypass control-gain set High

matching exit-flow BC's.

It was found that higher gain levels for the simulated air-bypass control could also yield good stability for the normal-shock with greater suppression of the main-frequency of pressure oscillations; however, undesirable high- 
frequency harmonics (Figure 17) would also occur with higher control-gains. Induced harmonic transients at highfrequencies with an over-active bypass control may increase the bleed flow and bypass flow, thereby reduce the thrust stream airflow to the engine and increase added drag from dumping the bleed flow overboard.

Some dynamic parameters in this simulation scheme that might need calibration (using system-tests data) are those in Part "d" (mentioned in Section I -- Introduction) to accurately model the effects of air bypass plenum dynamics on inlet exit-flow conditions or turbine-inlet couplings. Part "c" of the current simulation scheme could also be replaced by a dynamics model, such as by using an approximation of the oblique shocks and flow states in the front section of the HS Path as inputs to another LAPIN-type model simulating the HS Path into the diffuserisolator region. A turbine-engine simulation model could also be tied to this simulation via MMF to understand inlet-engine couplings.

If necessary, future work on this simulation can also include modeling the effects of structural compliances on inlet dynamics. Undesirable effects of structural vibrations on inlet dynamics that are due to servo-mechanics can be tuned-out using properly calibrated filters for actuator position feedback.

\section{Conclusion}

A simulation-framework using the Memory-Mapped-Files technique ${ }^{7,8}$ was implemented to tie together multiple computer models of sub-system dynamics to simulate inlet mode transition from turbine to ramjet-scramjet modes. The implemented simulation is applicable to a wide range of free-stream and exit-flow conditions. Preliminary simulation results show general agreements with CFD predictions ${ }^{10}$ of inlet mode-transition dynamics and stability. In addition, the simulation results show that inlet back-pressure dynamics is likely to be an important factor in mode-transition control. The simulation can be used for mode-transition control development once validated with system-characterization test data.

\section{Acknowledgments}

This simulation development for control-study of High-Speed Inlet Mode-Transition is funded by the NASA Hypersonic Project. The authors would like to express their appreciation for the technical guidance of Dr. James L. Pitman/LaRC (Principal Investigator, Hypersonic Project), Mr. Scott R. Thomas/GRC (CCE-LIMX Project Lead), Mr. John D. Saunders/GRC (CCE-LIMX Inlet-Research Lead), Mr. Donald I. Soloway/ARC (Associate PI/ GN\&C, Hypersonic Project); and Dr. Thomas J. Stueber/GRC (CCE-IMX Control-Research Lead). Last but not least, we wish to thank Mr. Paul Raitano/GRC for the wonderful scripting-tools he developed to facilitate the preparation of LAPIN run data.

\section{References}

${ }^{1}$ Saunders, J.D., Slater, J.W., Dippold, V., Lee, J., Sanders, B.W., and Weir, L.J., “Inlet Mode Transition Screening Test for a Turbine-Based Combined-Cycle Propulsion System,” JANNAF, May 2008.

${ }^{2}$ Stueber, T.J., Vrnak, D.R., Le, D.K., and Ouzts, P.J., “Control Activity in Support of NASA Turbine Based Combined Cycle (TBCC) Research,” NASA TM-2010-216109, March 2010.

${ }^{3}$ Sanders, B.W. and Weir, L.J., “Aerodynamic Design of a Dual-Flow Mach 7 Hypersonic Inlet System for a Turbine-Based Combined-Cycle Hypersonic Propulsion System”, NASA CR-2008-215214, June 2008.

${ }^{4}$ TechLand Research, Inc., Test Requirements for Combined-Cycle Engine (CCE) Large-Scale Inlet Mode Transition (LIMX) Test, NASA NRA Contract No. NNC08CA60C, September 2009.

${ }^{5}$ Varner, M.O., Martindale, W.R., Phares, W.J., Kneile, K.R., and Adams, Jr., J.C., "Large Perturbation Flow Field Analysis and Simulation for Supersonic Inlets,” NASA CR-174676, 1984.

${ }^{6}$ Martindale, W.R., et. al., "Large Perturbation Flow Field Analysis and Simulation for Supersonic Inlets—Program Modifications,” NAS3-24105 Task 2608, 1987.

${ }^{7}$ Vrnak, D.R., Stueber, T.J., Le, D.K., “A Novel Technique for Running the NASA Legacy Code LAPIN Synchronously With Simulations Developed Using Simulink,” NASA TM-2012-217444, July 2012.

${ }^{8}$ Gaudette, T., "Using Memory Mapped Files for Fast Data Transfer," MATLAB Digest - March 2004, The Mathworks, Inc.

${ }^{9}$ Sanders, B. W., Weir, L. J., Saunders, J. D., and Foster, L. E., "Mach 4 Experimental Demonstration of Inlet Mode Transition Technology for a Turbine-Based Combined Cycle Hypersonic Propulsion System,” NASA/TM-2012-217647; September 2012.

${ }^{10}$ Slater, J. W., and Saunders, J. D., “CFD Simulation of Hypersonic TBCC Inlet Mode Transition,” AIAA-2009-7349, 16th AIAA/DLR/DGLR International Space Planes and Hypersonic Systems and Technologies Conference, October 2009.

${ }^{11}$ Le, D.K., Owen A.K., Simon, D.L., "Wavelet Analysis of Air-Jet Injection Stall Control Effects in an Axi-Centrifugal GasTurbine Engine,” AIAA-97-2774, 33 ${ }^{\text {rd }}$ Joint Propulsion Conference, July 1997. 